\title{
2
}

\section{STRONGLY OMNIPRESENT INTEGRAL OPERATORS}

\author{
L. Bernal-González, M.C. Calderón-Moreno and \\ K.-G. Grosse-Erdmann*
}

An operator $T$ on the space $H(G)$ of holomorphic functions on a domain $G$ is strongly omnipresent whenever there is a residual set of functions $f \in$ $H(G)$ such that $T f$ exhibits an extremely "wild" behaviour near the boundary. The concept of strong omnipresence was recently introduced by the first two authors. In this paper it is proved that a large class of integral operators including Volterra operators with or without a perturbation by differential operators has this property, completing earlier work about differential and antidifferential operators.

\section{Introduction}

In a recent paper the first two authors have introduced and studied the notion of $T$-monsters and the related notion of strongly omnipresent operators, see [BC1]. These concepts were motivated by the idea of a holomorphic monster as defined by W. Luh [Lu1] and by a result of the third author [Gr1] that, in the sense of Baire categories, almost every holomorphic function is a monster; see also [Lu2], [Sch], [LMM] and [Gr2, Section 4b].

Let $T$ be a (not necessarily linear) operator on the space $H(G)$ of holomorphic functions on a domain $G$ in $\mathbf{C}$. Then, roughly speaking, a $T$-monster is a holomorphic function in $G$ whose image under $T$ has an extremely "wild" behaviour near every boundary point of $G$. Strongly omnipresent operators may be characterized as those operators $T$ for which almost every holomorphic function on $G$ is a $T$-monster. In another recent paper

\footnotetext{
${ }^{*}$ The work of the first two authors has been partially supported by DGES grant PB96-1348 and the Junta de Andalucía.
} 
the authors have extended the notions of $T$-monsters and strong omnipresence slightly by allowing the point of infinity as a boundary point of $G$, see [BCG].

Before we can give the formal definitions we need to fix some notation and terminology. By $\mathbf{D}$ we denote the open unit ball in $\mathbf{C}$. For a subset $A$ of $\mathbf{C}, \bar{A}$ represents the closure of $A, A^{0}$ its interior and $\partial A$ its boundary; in addition we set $\|f\|_{A}:=\sup |f(z)|$, where $f$ is a complex function defined on $A$. An affine linear transformation $\tau: \mathbf{C} \rightarrow \mathbf{C}$ is a mapping of the form $\tau(z)=a z+b$ with constants $a$ and $b$.

A closed ball $B=\{z \in \mathbf{C}:|z-a| \leq r\}$ is always assumed to have positive radius $r>0$. For such a ball $B$ and a point $b \in B$ we denote by $A(B)$ the space of all functions that are continuous in $B$ and holomorphic in $B^{0}$, and by $A_{b}(B)$ the subspace of $A(B)$ consisting of all functions with a zero at $b$. We endow $A(B)$ and $A_{b}(B)$ with the maximum norm $\|\cdot\|_{\infty}:=\|\cdot\|_{B}$.

Let $G$ be a domain in $\mathbf{C}$. Then $H(G)$ denotes the space of holomorphic functions on $G$, endowed with the usual topology of local uniform convergence. The boundary $\partial G$ will be taken in the extended complex plane $\mathbf{C}_{\infty}=\mathbf{C} \cup\{\infty\}$, where $\mathbf{C}_{\infty}$ is topologized by the chordal metric. We denote by $O(\partial G)=\left\{V \subset \mathbf{C}_{\infty}: V\right.$ is open and $\left.V \cap \partial G \neq \emptyset\right\}$ the set of all open subsets of $\mathbf{C}_{\infty}$ that meet the boundary of $G$.

In this paper, an operator always refers to a continuous (not necessarily linear) mapping. With this we can recall the following definitions from [BCG], see also [BC1].

Definition 1.1 Let $G$ be a domain in $\mathbf{C}$ and $T: H(G) \rightarrow H(G)$ an operator. Then a function $f \in H(G)$ is called a $T$-monster if for each $g \in H(\mathbf{D})$ and each $t \in \partial G$ there exists a sequence $\left(\tau_{n}\right)$ of affine linear transformations $\tau_{n}(z)=a_{n} z+b_{n}$ with

$$
\begin{gathered}
\tau_{n}(\mathbf{D}) \subset G \text { for all } n \in \mathbf{N} \text {, and } \\
\tau_{n}(z) \rightarrow t \quad(n \rightarrow \infty) \quad \text { uniformly on } \mathbf{D}
\end{gathered}
$$

such that

$$
(T f)\left(\tau_{n}(z)\right) \rightarrow g(z) \quad \text { as } \quad n \rightarrow \infty
$$

locally uniformly in $\mathbf{D}$. 
Thus, near any boundary point of $G$ the function $T f$ can approximate any given holomorphic function $g \in H(\mathbf{D})$ on suitable open balls $\tau(\mathbf{D})$. For brevity, if $A \subset \mathbf{C}$ we will write $L T(A)$ for the set of all affine linear transformations $\tau$ with $\tau(\mathbf{D}) \subset A$.

Definition 1.2 Let $G$ be a domain in C. Then an operator $T: H(G) \rightarrow H(G)$ is called strongly omnipresent if for all $g \in H(\mathbf{D}), \varepsilon>0, r \in(0,1)$ and $V \in O(\partial G)$ the set

$$
\begin{gathered}
U(T, g, \varepsilon, r, V):=\{f \in H(G): \text { there exists some } \tau \in L T(V \cap G) \\
\text { such that } \left.\|(T f) \circ \tau-g\|_{r \overline{\mathbf{D}}}<\varepsilon\right\}
\end{gathered}
$$

is dense in $H(G)$.

It turns out that an operator $T$ is strongly omnipresent if and only if the set of $T$-monsters is residual, that is, its complement in $H(G)$ is of first Baire category; in other words: if and only if almost every holomorphic function on $G$ is a $T$-monster. This can be accomplished by expressing the set of $T$-monsters as a suitable countable intersection of sets $U(T, g, \varepsilon, r, V)$, cf. [BC1, Theorem 2.2].

In [BCG, Section 2] the authors have derived conditions under which a general operator is strongly omnipresent. Examples of strongly omnipresent operators have been obtained in [BC1, Sections 3, 4] and [BCG, Sections 2, 3]. Specifically, if $\Phi(z)=\sum_{j=0}^{\infty} a_{j} z^{j}$ is a non-zero entire function of subexponential type then the associated linear differential operator $\Phi(D)=\sum_{j=0}^{\infty} a_{j} D^{j}$ on $H(G)$ is strongly omnipresent. Here $D$ is the differentiation operator $D f=f^{\prime}, D^{0}=I$ is the identity operator and $D^{j+1}=D \circ D^{j}$; and $\Phi$ is said to be of subexponential type if for every $\varepsilon>0$ there is a constant $M=M(\varepsilon)>0$ such that

$$
|\Phi(z)| \leq M e^{\varepsilon|z|} \quad \text { for all } z \in \mathbf{C} .
$$

If $G \neq \mathbf{C}$ is a simply connected domain, $a \in G$ and $\Psi(z)=\sum_{j=0}^{\infty} a_{j} z^{j}$ is a non-zero function that is holomorphic at 0 then the corresponding linear antidifferential operator $\Psi\left(D_{a}^{-1}\right)=$ $\sum_{j=0}^{\infty} a_{j} D_{a}^{-j}$ on $H(G)$ is strongly omnipresent. Here $D_{a}^{0}=I$ and, for $j \in \mathbf{N}, D_{a}^{-j} f$ denotes the unique antiderivative $F$ of $f$ of order $j$ such that $F^{(k)}(a)=0$ for $k=0,1, \ldots, j-1$. In fact, we have for $j \in \mathbf{N}$

$$
D_{a}^{-j} f(z)=\int_{a}^{z} f(t) \frac{(z-t)^{j-1}}{(j-1) !} d t \quad(z \in G) .
$$


Furthermore, the strongly omnipresent (left- or right-) composition operators and multiplication operators have been completely characterized. In the latter case the exact condition is that the multiplication function $\psi$ be non-identically zero on $G$.

From now on, $G$ will be a simply connected domain, $a \in G$ a fixed point and $\varphi: G \times G \rightarrow \mathbf{C}$ a function that is holomorphic in both variables.

Our aim in this paper is to study the strong omnipresence of operators $T: H(G) \rightarrow H(G)$ of the form

$$
T f(z)=S f(z)+\int_{a}^{z} f(t) \varphi(z, t) d t \quad(z \in G),
$$

where $S: H(G) \rightarrow H(G)$ is another (in general non-integral) operator. In Section 2 we obtain a general sufficient condition for $T$ to be strongly omnipresent. In Section 3 we apply this result to show, among other things, that the sum of a Volterra operator and a finite order differential operator with holomorphic coefficients is always strongly omnipresent. It follows, in particular, that every Volterra operator of the first or second kind is strongly omnipresent, thus generalizing earlier work in [BC1]. Let us emphasize that while in [BCG] Runge's theorem was sufficient for approximations, Mergelyan's theorem will be crucial for the results in the present paper.

\section{A sufficient condition}

In $[\mathrm{BC} 1$, Theorem 4.2] the first two authors proved that the Volterra operator of the first or second kind on $H(G)$ given by

$$
T f(z)=\lambda f(z)+\int_{a}^{z} f(t) h(z-t) d t
$$

is strongly omnipresent, where $\lambda \in \mathbf{C}, h$ is a non-zero entire function of exponential type and the integral is taken along any rectifiable curve in $G$ joining $a$ with $z$.

The proof of this result has two well-distinguished steps, and the restriction on the integral kernel of $T$ to be a convolution kernel is only necessary in one of them. If we take this into account then a careful study of the proof enables us to obtain a sufficient condition for the strong omnipresence of integral operators with general kernels. Moreover, we can replace the operator of multiplication with $\lambda$ by more general operators $S$. 
Theorem 2.1 Let $S: H(G) \rightarrow H(G)$ be an operator. Then the operator $T: H(G) \rightarrow H(G)$ defined by

$$
T f(z)=S f(z)+\int_{a}^{z} f(t) \varphi(z, t) d t \quad(z \in G)
$$

is strongly omnipresent if for each $V \in O(\partial G)$ there are closed balls $B, B^{\prime}$ in $V \cap G$ with $B^{\prime} \subset B$ and a point $b \in \partial B$ such that

(a) the operator $S$ extends continuously to an operator

$$
\widetilde{S}: A(B) \rightarrow A\left(B^{\prime}\right),
$$

(b) the operator $\widetilde{T}: A_{b}(B) \rightarrow A\left(B^{\prime}\right)$ defined by

$$
\widetilde{T} f(z)=\widetilde{S} f(z)+\int_{b}^{z} f(t) \varphi(z, t) d t \quad\left(z \in B^{\prime}\right)
$$

has dense range.

Proof. The proof is an adaptation of the first half of the proof of Theorem 4.2 in [BC1]. We fix $g \in H(\mathbf{D}), \varepsilon>0, r \in(0,1)$ and $V \in O(\partial G)$, where we can assume that $g$ is a polynomial. We then have to show that $U(T, g, \varepsilon, r, V)$ is dense in $H(G)$. To see this, fix a basic open subset

$$
D\left(h, K, \varepsilon_{1}\right):=\left\{f \in H(G):\|f-h\|_{K}<\varepsilon_{1}\right\}
$$

of $H(G)$, where $K$ is a compact subset of $G, h \in H(G)$ and $\varepsilon_{1}>0$. Then we can find

- a compact subset $L$ of $G$ with connected complement that contains $a$ and $K$,

- closed balls $B, B^{\prime}$ in $V \cap G$ with $B^{\prime} \subset B$ and a point $b \in \partial B$ that satisfy the hypothesis in the statement of the theorem; by starting with a smaller set $V$, if necessary, we can assume that $B \cap L=\emptyset$,

- an affine linear transformation $\tau$ with $\tau(\overline{\mathbf{D}})=B^{\prime}$, and

- a rectifiable Jordan arc $\gamma$ joining $a$ and $b$ in $G$ such that $\gamma \cap B=\{b\}$ and such that $L \cup \gamma \cup B$ has connected complement. 
Let $h_{1}$ be a function that is continuous on $L \cup \gamma$, agrees with $h$ on $L$ and satisfies $h_{1}(b)=0$. It follows from (b) that there is function $h_{2} \in A_{b}(B)$ such that

$$
\left|\left(\widetilde{S} h_{2}(z)+\int_{b}^{z} h_{2}(t) \varphi(z, t) d t\right)-\left(g\left(\tau^{-1}(z)\right)-\int_{\gamma} h_{1}(t) \varphi(z, t) d t\right)\right|<\varepsilon \quad\left(z \in B^{\prime}\right) .
$$

By (a) the operator $\widetilde{S}: A(B) \rightarrow A\left(B^{\prime}\right)$ is continuous, and the same is obviously true for the operators $A(B) \rightarrow A\left(B^{\prime}\right), \psi \mapsto \int_{b}^{\cdot} \psi(t) \varphi(\cdot, t) d t$ and $A(\gamma) \rightarrow A\left(B^{\prime}\right), \psi \mapsto \int_{\gamma} \psi(t) \varphi(\cdot, t) d t$. Hence, by (2), there exists a $\delta>0$ such that if $f \in H(G)$ satisfies

$$
\left|f(z)-h_{1}(z)\right|<\delta \quad \text { for } \quad z \in \gamma
$$

and

$$
\left|f(z)-h_{2}(z)\right|<\delta \quad \text { for } \quad z \in B
$$

then

$$
\left|\left(\widetilde{S} f(z)+\int_{b}^{z} f(t) \varphi(z, t) d t\right)-\left(g\left(\tau^{-1}(z)\right)-\int_{\gamma} f(t) \varphi(z, t) d t\right)\right|<\varepsilon \quad\left(z \in B^{\prime}\right) .
$$

Now, since the function that agrees with $h_{1}$ on $L \cup \gamma$ and with $h_{2}$ on $B$ is continuous on $L_{0}:=L \cup \gamma \cup B$ (note that $h_{1}(b)=h_{2}(b)=0$ ) and holomorphic in the interior of $L_{0}$, and since $L_{0}$ has connected complement it follows from Mergelyan's Theorem (see [Rud, Chapter 20]) that there exists a function $f \in H(G)$ that satisfies (3), (4) and

$$
\left|f(z)-h_{1}(z)\right|<\varepsilon_{1} \quad \text { for } z \in L
$$

Then $f$ also satisfies (5), which can be rewritten as

$$
\left|T f(z)-g\left(\tau^{-1}(z)\right)\right|<\varepsilon \quad\left(z \in B^{\prime}\right) .
$$

This shows that $f \in U(T, g, \varepsilon, r, V)$; note that $\tau(\overline{\mathbf{D}})=B^{\prime}$. In addition it follows from (6) that $f \in D\left(h, K, \varepsilon_{1}\right)$; recall that $h_{1}=h$ on $L \supset K$. Thus we have proved that

$$
U(T, g, \varepsilon, r, V) \cap D\left(h, K, \varepsilon_{1}\right) \neq \emptyset,
$$

as required.

Using standard arguments, condition (a) in the theorem is implied by the following stronger condition: 
(a') for every $\varepsilon>0$ there exists a $\delta>0$ such that for all $f, g \in H(G)$ we have that $\|f-g\|_{B}<\delta$ implies $\|S f-S g\|_{B^{\prime}}<\varepsilon$.

Moreover, if $S$ is a linear operator then the conditions (a) and (a') are in fact equivalent. This shows that condition (a) is related to the notion of local stability introduced by the authors in $[\mathrm{BCG}]$. For the sake of completeness, we provide the corresponding definition given in that paper. An operator $T$ is said to be locally stable near $\partial G$ if for each compact subset $K$ of $G$ there exists a compact subset $M$ of $G$ such that for each closed ball $B \subset G \backslash M$, each $f \in H(G)$ and each $\varepsilon>0$ there exist a closed ball $B^{\prime} \subset G \backslash K$ and $\delta>0$ such that if $g \in H(G)$ and $\|f-g\|_{B^{\prime}}<\delta$ then $\|T f-T g\|_{B}<\varepsilon$. In addition, we say that $T$ has locally dense range near $\partial G$ if there exists a compact subset $M$ of $G$ such that for each open ball $U \subset G \backslash M$, the operator $\left.f \in H(G) \mapsto(T f)\right|_{U} \in H(U)$ has dense range. Thus, while in [BCG, Section 2] we inferred strong omnipresence of an operator $T$ from local stability and local density of $T$ itself we ask here, roughly, for local stability and local density of two different operators that are related to $T$.

Based on Theorem 2.1 we can now show that several concrete operators are strongly omnipresent.

\section{Strongly omnipresent integral operators}

In the first result of this section we show that the restriction on the kernel of the Volterra operator (1) to be a convolution kernel as considered in [BC1] is not needed for strong omnipresence. Also, we can replace multiplication with a constant $\lambda \in \mathbf{C}$ by multiplication with a general holomorphic function $\psi \in H(G)$. We first study the case where $\psi$ is not identically zero.

Theorem 3.1 Assume that $\psi \in H(G)$ is non-zero. Then the operator $T: H(G) \rightarrow H(G)$ defined by

$$
T f(z)=\psi(z) f(z)+\int_{a}^{z} f(t) \varphi(z, t) d t \quad(z \in G)
$$

is strongly omnipresent.

Proof. We will show that the following assertion holds: 
(*) For every closed ball $B \subset G$ with $\psi(z) \neq 0$ for all $z \in B$ and every point $b \in \partial B$ the operator $\widetilde{T}: A_{b}(B) \rightarrow A_{b}(B)$ given by

$$
\widetilde{T} f(z)=\psi(z) f(z)+\int_{b}^{z} f(t) \varphi(z, t) d t \quad(z \in B)
$$

is onto.

Since, as is easy to see, $A_{b}(B)$ is dense in $A\left(B^{\prime}\right)$ for any closed ball $B^{\prime}$ contained in the interior of $B$ it then follows from Theorem 2.1 that $T$ is strongly omnipresent.

Now, since $\psi(z) \neq 0$ for all $z \in B$, we need only prove that the operator $T_{1}$ : $A_{b}(B) \rightarrow A_{b}(B)$ defined by

$$
T_{1} f(z)=f(z)+\int_{b}^{z} f(t) \varphi_{1}(z, t) d t \quad(z \in B)
$$

is onto, where $\varphi_{1}(z, t)=\frac{\varphi(z, t)}{\psi(z)}$, and this in turn is satisfied whenever $T_{1}$ is invertible. After rotation, translation, normalization and change of sign we can suppose that $B=\overline{\mathbf{D}}, b=1$ and that $T_{1}=I-K$, where $K$ is the operator

$$
K f(z)=\int_{1}^{z} f(t) \varphi_{1}(z, t) d t .
$$

We have that $T_{1}$ is invertible on $A_{1}(\overline{\mathbf{D}})$ if and only if $0 \notin \sigma\left(T_{1}\right)$, the spectrum of $T_{1}$. Hence, it suffices to demonstrate that $\sigma\left(T_{1}\right)=\{1\}$, which, in turn, is equivalent to $\sigma(K)=\{0\}$ (that is, that $K$ is quasi-nilpotent). By Gelfand's formula for the spectral radius we need to show that

$$
\left\|K^{n}\right\|^{1 / n} \rightarrow 0 \quad(n \rightarrow \infty)
$$

where $\|K\|:=\sup \left\{\|K f\|_{\infty}:\|f\|_{\infty} \leq 1\right\}$, the norm in the space of linear operators on $A_{1}(\overline{\mathbf{D}})$.

Now, (7) follows as in the classical case of Volterra operators on the real line. Let $f \in A_{1}(\overline{\mathbf{D}})$ with $\|f\|_{\infty} \leq 1$ and set $M:=\sup \left\{\left|\varphi_{1}(z, t)\right|:(z, t) \in \overline{\mathbf{D}} \times \overline{\mathbf{D}}\right\}$. Then it is clear that, for each $z \in \overline{\mathbf{D}}$,

$$
|K f(z)| \leq M|z-1|
$$

By iteration we obtain

$$
K^{2} f(z)=\int_{1}^{z}(K f)(t) \varphi_{1}(z, t) d t
$$




$$
=\int_{0}^{1}(K f)(1-u+z u) \varphi_{1}(z, 1-u+z u)(z-1) d u
$$

SO

$$
\begin{gathered}
\left|K^{2} f(z)\right| \leq \int_{0}^{1} M|1-u+z u-1| \cdot M|z-1| d u \\
\leq M^{2}|z-1|^{2} \int_{0}^{1} u d u=\frac{M^{2}|z-1|^{2}}{2} .
\end{gathered}
$$

An induction procedure leads us to

$$
\left|K^{n} f(z)\right| \leq \frac{M^{n}|z-1|^{n}}{n !} \leq \frac{(2 M)^{n}}{n !} \quad\left(z \in \overline{\mathbf{D}},\|f\|_{\infty} \leq 1, n \in \mathbf{N}\right) .
$$

Then

$$
\left\|K^{n}\right\|^{1 / n} \leq \frac{2 M}{(n !)^{1 / n}} \rightarrow 0 \quad(n \rightarrow \infty) .
$$

Consequently, (7) is satisfied and the proof is finished.

We next want to study the operator $T$ given in Theorem 3.1 when $\psi=0$. For this we need the following lemma.

Lemma 3.2 Let $\varphi: G \times G \rightarrow \mathbf{C},(z, t) \mapsto \varphi(z, t)$ be a function that is holomorphic with respect to both variables such that for each number $n \in \mathbf{N}_{0}$ and each $w \in G$,

$$
\frac{\partial^{n} \varphi}{\partial z^{n}}(w, w)=0
$$

Then $\varphi$ is identically zero on $G \times G$.

Proof. It follows from the hypothesis that for every $w \in G$ the function

$$
z \mapsto \varphi(z, w)
$$

is holomorphic on $G$ and has vanishing derivatives of all orders $n \geq 0$ at $w$. Hence it must be identically zero on $G$, which implies the result since $w \in G$ is arbitrary.

With this we can complete Theorem 3.1 by considering the case $\psi=0$. Of course, we now have to assume that $\varphi \neq 0$. 
Theorem 3.3 Assume that $\varphi \neq 0$. Then the operator $T: H(G) \rightarrow H(G)$ defined by

$$
T f(z)=\int_{a}^{z} f(t) \varphi(z, t) d t \quad(z \in G)
$$

is strongly omnipresent.

Proof. We will show that the assumptions of Theorem 2.1 are satisfied with $S=0$. Thus let $V \in O(\partial G)$.

Since $\varphi$ is non-zero it cannot be identically zero on $(V \cap G) \times(V \cap G)$. Hence there is, by Lemma 3.2, a number $n \in \mathbf{N}_{0}$ for which there exists some $w \in V \cap G$ with

$$
\frac{\partial^{n} \varphi}{\partial z^{n}}(w, w) \neq 0
$$

Let $N$ be the least such number $n$. Then there exists a closed ball $B \subset V \cap G$ such that, for all $w \in B$,

$$
\frac{\partial^{n} \varphi}{\partial z^{n}}(w, w)=0 \quad(n=0,1, \ldots, N-1)
$$

and

$$
\frac{\partial^{N} \varphi}{\partial z^{N}}(w, w) \neq 0
$$

We fix a point $b \in \partial B$ and consider the operator $\widetilde{T}: A_{b}(B) \rightarrow A(B)$ defined by

$$
\widetilde{T} f(z)=\int_{b}^{z} f(t) \varphi(z, t) d t \quad(z \in B)
$$

Since the linear combinations of $(z-b)^{m}, m \geq N+2$, are dense in $H\left(B^{0}\right)$ and hence in $A\left(B^{\prime}\right)$ for any closed ball $B^{\prime} \subset B^{0}$ we see that the assumptions of Theorem 2.1 are satisfied if for every $m \geq N+2$ the equation

$$
\widetilde{T} f(z)=(z-b)^{m} \quad(z \in B)
$$

has a solution in $A_{b}(B)$. It will then follow that $T$ is strongly omnipresent.

Now, it is a simple consequence of (8) that for each $f \in A_{b}(B)$ the function $\widetilde{T} f$ is $(N+1)$-times continuously differentiable on $B$ with

$$
D^{n}(\widetilde{T} f)(w)=\int_{b}^{w} f(t) \frac{\partial^{n} \varphi}{\partial z^{n}}(w, t) d t \quad(n=0,1, \ldots, N)
$$

and

$$
D^{N+1}(\widetilde{T} f)(w)=f(w) \frac{\partial^{N} \varphi}{\partial z^{N}}(w, w)+\int_{b}^{w} f(t) \frac{\partial^{N+1} \varphi}{\partial z^{N+1}}(w, t) d t
$$


for all $w \in B$. It follows from (9) and assertion $(*)$ in the proof of Theorem 3.1, taking $\psi(w)=\frac{\partial^{N} \varphi}{\partial z^{N}}(w, w)$, that for each $m \geq N+2$ there exists a function $f \in A_{b}(B)$ such that

$$
D^{N+1}(\widetilde{T} f)(w)=D^{N+1}\left((w-b)^{m}\right) \quad(w \in B) .
$$

Hence, $\widetilde{T} f$ and $(w-b)^{m}$ differ on $B$ by at most a polynomial $P(w)=\sum_{n=0}^{N} a_{n}(w-b)^{n}$, and it follows from (11) that, indeed, $P=0$. This implies (10) and the proof is finished.

Using the above results we can in fact show that the multiplication operator in Theorem 3.1 can be replaced, more generally, by linear combinations of operators of the form

$$
f \in H(G) \mapsto \psi(z) D^{n} f(z) \in H(G),
$$

where $n \in \mathbf{N}_{0}$ and $\psi \in H(G)$.

Theorem 3.4 Let $a_{n}(n=0, \ldots, N)$ be holomorphic functions in $G$, where $a_{N}$ is not identically zero. Then the operator $T: H(G) \rightarrow H(G)$ defined by

$$
T f(z)=\sum_{n=0}^{N} a_{n}(z)\left(D^{n} f\right)(z)+\int_{a}^{z} f(t) \varphi(z, t) d t \quad(z \in G)
$$

is strongly omnipresent.

Proof. Let $B$ and $B^{\prime}$ be closed balls in $G$ with $B^{\prime} \subset B^{0}$ and $b \in \partial B$ a fixed point. Since the operator $S$ given by $S f=\sum_{n=0}^{N} a_{n}\left(D^{n} f\right)$ clearly satisfies condition (a) of Theorem 2.1 it suffices to show that whenever $a_{N}(z) \neq 0$ for all $z \in B$ the operator $\widetilde{T}: A_{b}^{N}(B) \rightarrow A\left(B^{\prime}\right)$ defined by

$$
\widetilde{T} f(z)=\sum_{n=0}^{N} a_{n}(z)\left(D^{n} f\right)(z)+\int_{b}^{z} f(t) \varphi(z, t) d t \quad\left(z \in B^{\prime}\right)
$$

has dense range, where $A_{b}^{N}(B)$ denotes the subspace of $A_{b}(B)$ consisting of all functions $f \in$ $A(B)$ that are $N$-times continuously differentiable in $B$ with $\left(D^{n} f\right)(b)=0$ for $n=0, \ldots, N$.

First, let $\psi: G \times G \rightarrow \mathbf{C}$ be the unique holomorphic function of two variables such that for all $(z, t) \in G \times G$ we have

$$
\frac{\partial^{N} \psi}{\partial t^{N}}(z, t)=\varphi(z, t)
$$


and

$$
\frac{\partial^{n} \psi}{\partial t^{n}}(z, b)=0 \quad \text { for } \quad n=0, \ldots, N-1 .
$$

Indeed, $\psi(z, t)=\int_{b}^{t} \frac{(t-\zeta)^{N-1}}{(N-1) !} \varphi(z, \zeta) d \zeta$. Now $N$-fold integration by parts gives

$$
\begin{gathered}
\int_{b}^{z} f(\zeta) \varphi(z, \zeta) d \zeta=\int_{b}^{z} f(\zeta) \frac{\partial^{N} \psi}{\partial t^{N}}(z, \zeta) d \zeta= \\
=\sum_{n=0}^{N-1}(-1)^{n}\left(D^{n} f\right)(z) \frac{\partial^{N-1-n} \psi}{\partial t^{N-1-n}}(z, z)+(-1)^{N} \int_{b}^{z}\left(D^{N} f\right)(\zeta) \psi(z, \zeta) d \zeta .
\end{gathered}
$$

We can therefore write

$$
\widetilde{T} f(z)=a_{N}(z)\left(D^{N} f\right)(z)+\sum_{n=0}^{N-1} b_{n}(z)\left(D^{n} f\right)(z)+(-1)^{N} \int_{b}^{z}\left(D^{N} f\right)(t) \psi(z, t) d t
$$

with certain functions $b_{0}, \ldots, b_{N-1}$ that are holomorphic in $G$.

On the other hand, for $f \in A_{b}^{N}(B)$ we have $D^{n} f=D_{b}^{-N+n} D^{N} f$ for $n=0, \ldots, N-$ 1 , where $D_{b}^{-j}(j \in \mathbf{N})$ denotes the antiderivative operator of order $j$, cf. the Introduction. Hence,

$$
\sum_{n=0}^{N-1} b_{n}(z)\left(D^{n} f\right)(z)=\sum_{n=0}^{N-1} b_{n}(z)\left(D_{b}^{-N+n} D^{N} f\right)(z) .
$$

Since each operator $D_{b}^{-j}$ can be written as an integral operator,

$$
\left(D_{b}^{-j} f\right)(z)=\int_{b}^{z} f(t) \frac{(z-t)^{j-1}}{(j-1) !} d t
$$

we see from (12) and (13) that

$$
\widetilde{T} f(z)=a_{N}(z)\left(D^{N} f\right)(z)+\int_{b}^{z}\left(D^{N} f\right)(t) \widetilde{\psi}(z, t) d t
$$

with a holomorphic function of two variables $\widetilde{\psi}: G \times G \rightarrow \mathbf{C}$.

Let us now consider the operator $T_{1}: A_{b}(B) \rightarrow A\left(B^{\prime}\right)$ that is defined by

$$
T_{1} h(z)=a_{N}(z) h(z)+\int_{b}^{z} h(t) \widetilde{\psi}(z, t) d t .
$$

Since $a_{N}(z) \neq 0$ for all $z \in B$ by assumption it follows from assertion $(*)$ in the proof of Theorem 3.1 that $T_{1}$ has dense range. Since $D^{N}: A_{b}^{N}(B) \rightarrow A_{b}(B)$ is onto we see that also the operator $\widetilde{T}=T_{1} \circ D^{N}: A_{b}^{N}(B) \rightarrow A\left(B^{\prime}\right)$ has dense range, which had to be shown.

In particular, when all the holomorphic coefficients are constants we obtain the following. 
Corollary 3.5 Let $P$ be a non-zero polynomial. Then the operator $T: H(G) \rightarrow H(G)$ defined by

$$
T f(z)=P(D) f(z)+\int_{a}^{z} f(t) \varphi(z, t) d t \quad(z \in G)
$$

is strongly omnipresent.

As an application of this result, let $\Psi(z)=\sum_{j=0}^{\infty} a_{j} z^{j}$ be holomorphic at the origin. Then the antidifferential operator $\Psi\left(D_{a}^{-1}\right)$ as defined in the Introduction is a Volterra operator, cf. (1): just take $\lambda=a_{0}$ and $h(z)=\sum_{j=1}^{\infty} a_{j} \frac{z^{j-1}}{(j-1) !}$. The corollary then implies that a non-zero sum of a finite order differential operator $P(D)$ and an infinite order antidifferential operator is strongly omnipresent. In particular we see that Corollary 4.3 of [BC1], by which the operator $\Psi\left(D_{a}^{-1}\right)$ is strongly omnipresent whenever $\Psi \neq 0$ and $G \neq \mathbf{C}$, remains true for $G=\mathbf{C}$.

We are now going to show that we can consider infinite order differential operators $\Phi(D)$ instead of $P(D)$ when the integral operator is a finite order antidifferential operator.

Theorem 3.6 Let $\Phi$ be a non-zero entire function of subexponential type and $P$ be a polynomial. Then the operator $T: H(G) \rightarrow H(G)$ defined by

$$
T f(z)=\Phi(D) f(z)+P\left(D_{a}^{-1}\right) f(z) \quad(z \in G)
$$

is strongly omnipresent.

Proof. We fix closed balls $B, B^{\prime}$ in $G$ with $B^{\prime} \subset B^{0}$ and a point $b \in \partial B$. A simple application of Cauchy's inequalities shows that $\Phi(D)$ is a continuous linear operator on $H(O)$ for any open subset $O \subset \mathbf{C}$, in particular on $H\left(B^{0}\right)$. Hence $\Phi(D)$ always satisfies condition (a') at the end of Section 2 and hence also condition (a) of Theorem 2.1. Thus we only need to verify that condition (b) holds for the operator $\widetilde{T}: A_{b}(B) \rightarrow A\left(B^{\prime}\right), \widetilde{T}=\Phi(D)+P\left(D_{b}^{-1}\right)$.

Suppose that $\Phi(z)=\sum_{n=0}^{\infty} a_{n} z_{n}$ and $P(z)=\sum_{n=0}^{N} p_{n} z_{n}$. We then consider the entire function $\Phi_{1}$ of subexponential type given by

$$
\Phi_{1}(z)=\sum_{n=0}^{\infty} c_{n} z^{n}=\sum_{n=0}^{\infty} a_{n} z^{n+N}+\sum_{n=0}^{N} p_{N-n} z^{n} .
$$

It is clear that

$$
\widetilde{T}=\Phi_{1}(D) \circ D_{b}^{-N} .
$$


In order to show that $\widetilde{T}: A_{b}(B) \rightarrow A\left(B^{\prime}\right)$ has dense range let $h \in A\left(B^{\prime}\right)$ and $\varepsilon>0$. Then there exists a polynomial $Q$ such that

$$
\|h-Q\|_{B^{\prime}}<\varepsilon
$$

Since $\Phi_{1}(D)$ is a surjective operator on $H(\mathbf{C})$ by [Ehr] or [Mal] there is an entire function $f_{1}$ with

$$
Q=\Phi_{1}(D) f_{1}
$$

Now, the entire functions that have a zero of multiplicity at least $N+1$ at $b$ are dense in $H\left(B^{0}\right)$ and the operator $\Phi_{1}(D): H\left(B^{0}\right) \rightarrow H\left(B^{0}\right)$ is continuous. Hence, by (14) and (15), there is an entire function $f_{2}$ that has a zero of multiplicity at least $N+1$ at $b$ with

$$
\left\|h-\Phi_{1}(D) f_{2}\right\|_{B^{\prime}}<\varepsilon
$$

Since

$$
f_{2}=D_{b}^{-N} f \quad \text { with } \quad f:=D^{N} f_{2}
$$

we have that $f \in A_{b}(B)$ and

$$
\|h-\widetilde{T} f\|_{B^{\prime}}=\left\|h-\Phi_{1}(D) \circ D_{b}^{-N} f\right\|_{B^{\prime}}=\left\|h-\Phi_{1}(D) f_{2}\right\|_{B^{\prime}}<\varepsilon,
$$

so that $\widetilde{T}: A_{b}(B) \rightarrow A\left(B^{\prime}\right)$ indeed has dense range, and the proof is finished.

From the last two results we know that the sum of an infinite (resp. finite) order differential operator and a finite (resp. infinite) order antidifferential operator is always strongly omnipresent. The general case where the two operators are of infinite order remains open. Up to date, we can only say that these operators always have the weaker property of omnipresence (see $[\mathrm{Ber}]$ ), as was recently proved by the first two authors [BC2] following another point of view.

\section{REFERENCES}

[Ber] L. Bernal-GonzÁLez, Omnipresent holomorphic operators and maximal cluster sets, Colloq. Math. 63 (1992), 315-322.

[BC1] L. Bernal-GonzÁlez and M.C. Calderón-Moreno, Holomorphic T-monsters and strongly omnipresent operators, J. Approx. Theory 104 (2000), 204-219. 
[BC2] L. Bernal-GonzÁlez and M.C. Calderón-Moreno, Operators with dense images everywhere, submitted.

[BCG] L. Bernal-González, M.C. Calderón-Moreno and K.-G. GrosseERDmann, Strongly omnipresent operators: general conditions and applications to composition operators, submitted.

[Ehr] L. Ehrenpreis, Mean periodic functions I, Amer. J. Math. 77 (1955), 293-328.

[Gr1] K.-G. Grosse-Erdmann, Holomorphe Monster und universelle Funktionen, Mitt. Math. Sem. Giessen 176 (1987).

[Gr2] K.-G. Grosse-Erdmann, Universal families and hypercyclic operators, Bull. Amer. Math. Soc. (N.S.) 36 (1999), 345-381.

[Lu1] W. LuH, Holomorphic monsters, J. Approx. Theory 53 (1988), 128-144.

[Lu2] W. LuH, Multiply universal holomorphic functions, J. Approx. Theory 89 (1997), $135-155$.

[LMM] W. Luh, V.A. Martirosian And J. Müller, T-universal functions with lacunary power series, Acta Sci. Math. (Szeged) 64 (1998), 67-79.

[Mal] B. MALGRANGE, Existence et approximation des solutions des équations aux dérivées partielles et des équations de convolution, Ann. Institut Fourier (Grenoble) 6 (1955/1956), 271-355.

[Rud] W. Rudin, Real and Complex Analysis, 3rd edition, McGraw-Hill, New York, 1987.

[Sch] I. SCHNEIDER, Schlichte Funktionen mit universellen Approximationseigenschaften, Mitt. Math. Sem. Giessen 230 (1997).

Luis Bernal González

Departamento de Análisis Matemático

Facultad de Matemáticas, Apdo. 1160

Avenida Reina Mercedes

41080 Sevilla, Spain

e-mail: lbernal@cica.es

Karl-Goswin Grosse-Erdmann

Fachbereich Mathematik

FernUniversität Hagen

58084 Hagen, Germany

e-mail: kg.grosse-erdmann@fernuni-hagen.de

\author{
María del Carmen Calderón Moreno \\ Departamento de Análisis Matemático \\ Facultad de Matemáticas, Apdo. 1160 \\ Avenida Reina Mercedes \\ 41080 Sevilla, Spain \\ e-mail: mccm@cica.es
}

2000 Mathematics Subject Classification: primary 30E10, 47G10; secondary 30H05, 46E10, $47 \mathrm{E} 05$. 